Document downloaded from:

http://hdl.handle.net/10251/115305

This paper must be cited as:

Tarrazó-Serrano, D.; Castiñeira Ibáñez, S.; Candelas Valiente, P.; Fuster Escuder, JM.; Rubio Michavila, C. (2019). Numerical simulation and laboratory measurements on the influence of fractal dimension on the acoustic beam modulation of a Polyadic Cantor Fractal lenses. Applied Acoustics. 148:119-122. doi:10.1016/j.apacoust.2018.12.012

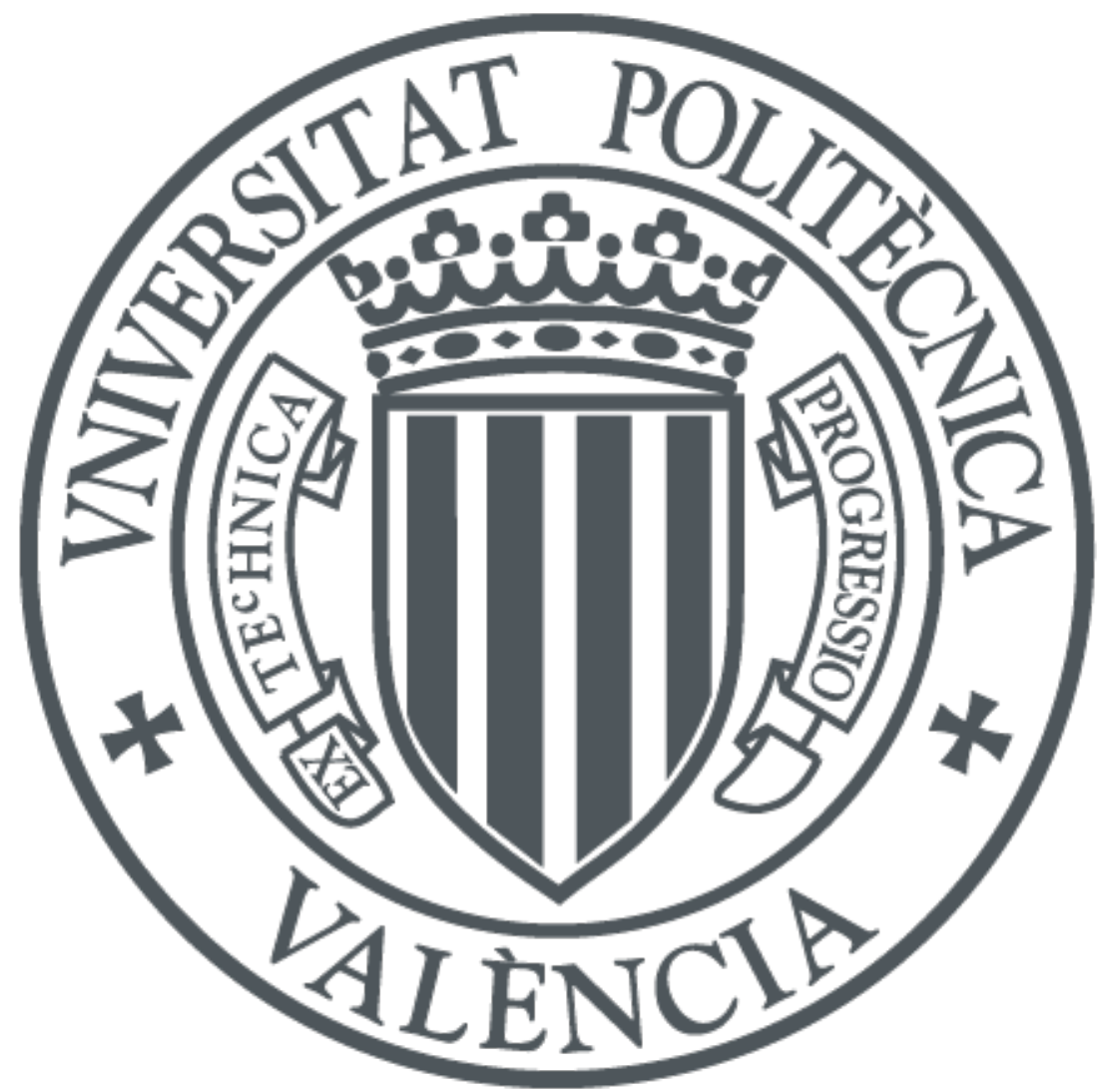

The final publication is available at

https://doi.org/10.1016/j.apacoust.2018.12.012

Copyright Elsevier

Additional Information 


\title{
Numerical simulation and laboratory measurements on the influence of fractal dimension on the acoustic beam modulation of a Polyadic Cantor Fractal lenses.
}

\author{
Daniel Tarrazó-Serrano ${ }^{a}$, Sergio Castiñeira-Ibáñez ${ }^{\mathrm{b}}$, Pilar Candelas $^{\mathrm{a}}$, José Miguel Fuster ${ }^{\mathrm{c}}$, Constanza \\ Rubio $^{\mathrm{a}, *}$ \\ ${ }^{a}$ Centro de Tecnologías Físicas, Universitat Politècnica de València, Camí de Vera s/n, València, 46022, Spain \\ ${ }^{b}$ Departamento de Física Aplicada, Universitat Politècnica de València, Camí de Vera s/n, València, 46022, Spain \\ ${ }^{c}$ Departamento de Comunicaciones, Universitat Politècnica de València, Camí de Vera s/n, València, 46022, Spain
}

\begin{abstract}
The possibility of modulating the ultrasound beam produced by a transducer through lenses has become an important task. The fact that these lenses are flat, facilitates their design, construction and applications. If, in addition, the design itself incorporates geometries that affect differently the foci profile on the axial axis of the lens, improvements become more significant. In this work the design of a flat lens based on a Polyadic Cantor fractal geometries is presented. The influence of the so-called fractal dimension on the modulation of the ultrasonic beam to obtain the foci is analysed. In this paper, experimental results under controlled conditions are presented. The numerical solutions obtained have been validated.
\end{abstract}

Keywords: polyadic cantor sets, fractal, lenses, ultrasounds

\section{Introduction}

In many fields of science, the ability to modulate energy beams is a requirement. Due to its potential applications, one way to passively modulate the wave front and therefore the energy, are lenses which achieve this objective using different physics phenomena. Based on geometric optics, lenses use concave-convex surfaces to focus energy [1]. Another type of lens based on structures such as periodic systems or cylinders have also been developed $[2,3]$. In the last years, another type of lenses with flat faces have been developed. They achieve the same effects as conventional lenses [4-6]. In the acoustic field, specifically in ultrasounds, flat lenses allow the focusing and modulation of the pressure beams facilitat-

\footnotetext{
*Corresponding author.

Email address: crubiom@fis.upv.es (Constanza Rubio)
}

ing the use of different transducers $[7,8]$. Fractal geometries are suitable for modulating and focusing beams. These geometries have been used in several fields of science and engineering in order to describe some phenomena that occur in these areas $[9,10]$. In optics and electromagnetism, lenses based on fractal geometries have been implemented due to their self-similarity focusing capabilities [11-13]. In acoustics, fractal distribution of rigid cylinders embedded in air enabled higher attenuation bands than a periodic distribution $[14,15]$. In this work, planar lenses based on fractal geometries are presented. Numerical models have been validated by experimental measurements in controlled conditions. A fractal structure based on a Cantor set is constructed by splitting the initial segment in several identical parts. The ratio between the initial segment and the number of split parts is called scaling factor $(r)$. When this ratio is between 0 and $1 / 2$, this struc- 
ture is denominated Generalized Polyadic Cantor Sets (GPCS). The nature of the Cantor set is maintained but different fractal dimensions $(D)$ are obtained and these lenses are called Polyadic Cantor Fractal Lenses (PCFL)[16].

The paper is organized as follows: First, we explain the numerical model (FEM) to the case analysed here. Afterwards, the experimental setup used to validate the numerical results is presented. Numerical results as well as the comparison with the experimental ones are analysed and discussed in the next section. Finally, the last section contains the concluding remarks, where the results are summarized.

\section{Material and methods}

Before designing the lenses, implementation limitations must be taken into account. First, mechanical construction limitations have to be considered. The number of times that the initial segment is split (stage, s) is other limitation due to the small slit that can not be milled. It can also be noted that for the diffraction phenomenon to occur, sizes comparable to the wavelength, $\lambda$, must be considered. It can be observed in Fig.1a a fractal at stage 2 with central gap $\left(g_{\mathrm{c}}\right)$ value of $g_{c}=0.4(1-4 r)$, where $\mathrm{r}$ is the scaling factor [17]. The gap between blocks of elements when the stage is increased is called $\varepsilon$. In this figure, the complete set of fractal elements obtained is shown. The lens can be obtained with a rotation of $\pi$ on the axis normal to the XR plane (Fig. 1b).

Fractal dimension, D, can be understood as a measure of the space-filling capacity of a pattern that reveals how a fractal scales differently from the space it is embedded in and it is given by:

$$
D=-\frac{\ln 4}{\ln r}
$$

In this work, the influence of $D$ in three different PCFLs and their corresponding beam conformations is presented. For manufacturing reasons, the values of $\mathrm{D}$ chosen were $D=0.80,0.85$ and 0.90. Lenses have been mechanized by using 1 mm thick brass plates. An example of the lenses implemented for the experimental measurements is shown in Fig. 1c. The radius of the lens $(R)$ is $12 \mathrm{~cm}$.

The commercial software COMSOL Multiphysics is used to study the physical phenomena involved in the interaction between the ultrasound waves and the PCFL immersed in water, by means Finite Element Method (FEM) [18]. For this purpose, it is necessary to define the geometry, discretization the domain and solve the Helmholtz given in equation (2).

$$
\nabla\left(-\frac{1}{\rho} \nabla p\right)=\frac{\omega^{2}}{\rho c^{2}} p
$$

Where the water density $(\rho)$ is $1000 \mathrm{~kg} / \mathrm{m}^{3}$ and the sound propagation velocity, c, is $1500 \mathrm{~m} / \mathrm{s}$. The working frequency $(f)$ of the PCFLs is 250 $\mathrm{kHz}$ and the angular frequency $(\omega=2 \pi f)$. Finally, $p$ represents the acoustic pressure.

As it has been presented in previous works [19, 20], the following boundary conditions have been defined: the PCFL is considered infinitely rigid marking the contours of the elements applying the Newmann condition (the sound velocity in the contour is zero). The contours of the model are defined as wave radiation condition boundaries to emulate an infinitely large medium and therefore the Sommerfeld condition is satisfied. The axis of rotation is defined as axis of axisymmetry to simulate the $2 \pi$ rotation that makes up the PCFL in 3D. In Fig. 2a, the scheme of the model implemented in FEM is shown.

Lenses have been measured by using an immersion ultrasound high precision system provided by the Centro de Tecnologías Físicas at the Universitat Politècnica de València in order to validate and compare the proposed models as explained in previous works [21].

The measurement system is composed by a fixed emitter and a hydrophone coupled to the robotic system. This system obtains reliable and precise results that allow evaluating the acoustic phenomena involved in this type of lenses. As shown in Fig. 2b, the lens is immersed in a tank of distilled water. 


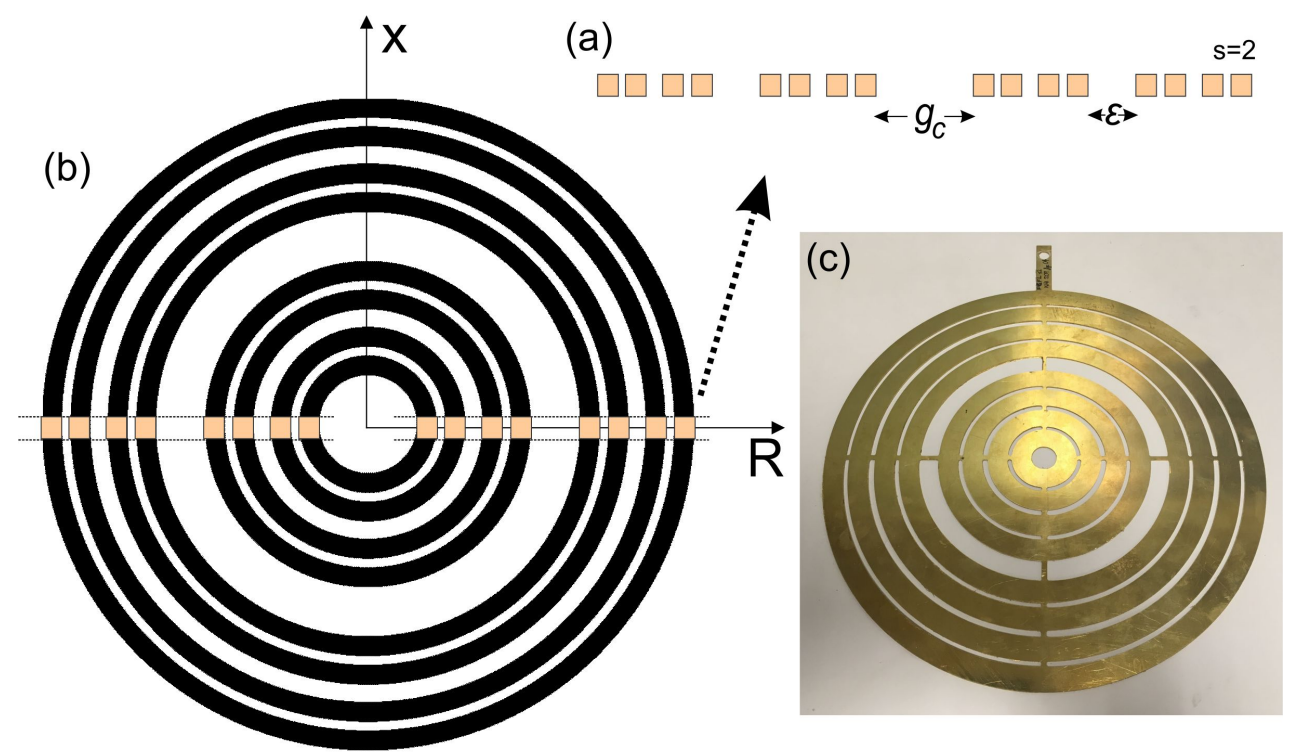

Figure 1: Schematic section diagram of the generation for (a) Polyadic Cantor Fractal Lens (PCFL) with stage 2 ( $\mathrm{s}=2$ ). The $g_{\mathrm{c}}$ is the width of the central gap and $\varepsilon$ is the width of lateral gap. (b) Generation of the PCFL. (c) PCFL lens considered and implemented in brass.
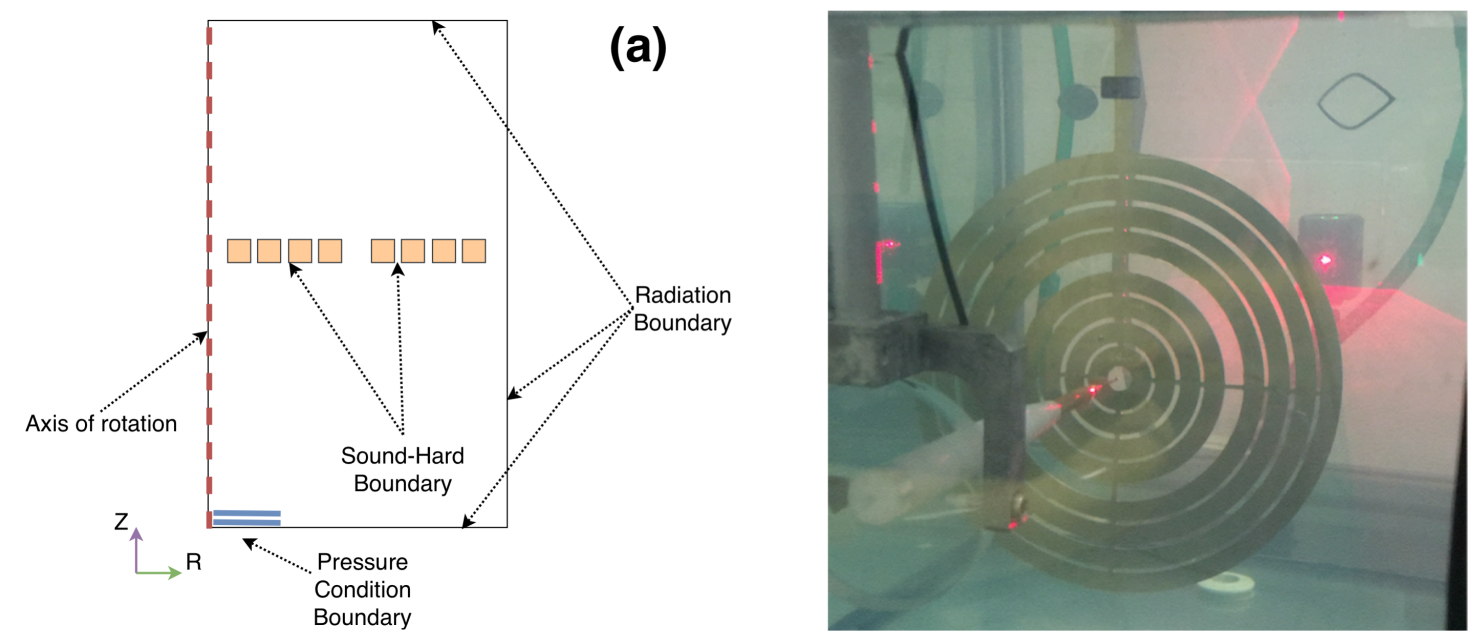

(b)

Figure 2: (a) Schematic diagram of the configuration simulated in the numerical domain where the solution are obtained. (b) Experimental Set-up

A plane immersion piston transducer built by Imasonic with $250 \mathrm{kHz}$ of central working frequency and an active diameter of $32 \mathrm{~mm}$ has been used as emitter. The hydrophone is a Polyvinylidene fluoride (PVDF) needle transducer (model MPM1/1, Precision Acoustic Ltd.) and has a diameter of $1.5 \mathrm{~mm}$ with a flat transfer function between 0.2 and $15 \mathrm{MHz} \pm 4 \mathrm{~dB}$. It has been used as receiver. Acoustic waves emitted by the piston transducer are detected by the needle hy- drophone. The pre-amplifier is used to amplify with low noise the hydrophone input signal before it is digitalized by the oscilloscope. This PC oscilloscope is a digital Picoscope Model 3224 from Pico Technology Co. When the data acquired are received by the computer, a time domain averaging with 100 different measurements is realized. The acoustic plane is obtained by automated scanning using steps of $1 \mathrm{x} 1 \mathrm{~mm}^{2}$. 

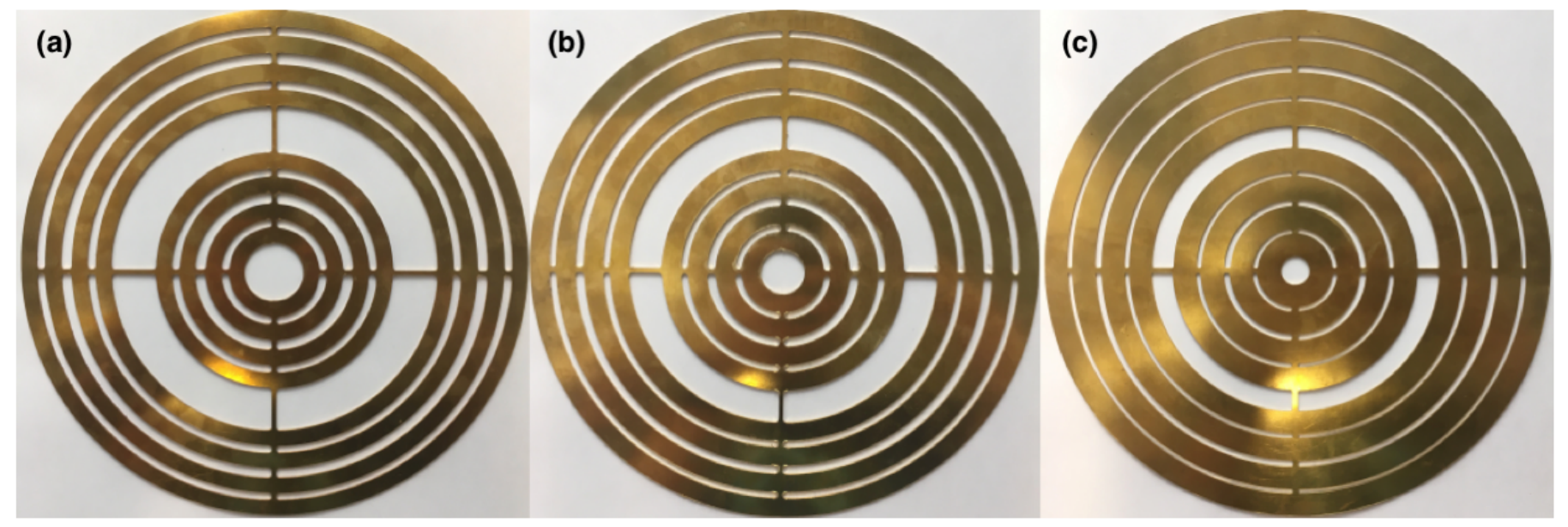

Figure 3: PCFLs implemented in brass (a) $\mathrm{D}=0.80$, (b) $\mathrm{D}=0.85$, (c) $\mathrm{D}=0.90$; It is observed that when the fractal dimension increases, the PCFL becomes more opaque.

\section{Results}

The influence of the fractal dimension parameter $\mathrm{D}$ on the modulation of the ultrasonic beam produced by the PCFL is analyzed. For this, a fractal dimension of value 0.80 is taken as starting point. The lens designed in metal with this fractal dimension is shown in the Fig. 3a, and Fig. 4a shows the numerical result obtained by the simulation model performed with FEM. It is observed that the main focus is located around $\mathrm{Z}$ $=8 \lambda$ of the center of the PCFL. This bulb has an absolute acoustic pressure in the order of $150 \%$ of the acoustic pressure emitted by the piston. Secondary foci are also seen near the PCFL, although these are of low power.

In the PCFL, two zones can be distinguished that will influence the modulation of the ultrasonic beam. These are the central hole, which is controlled by the central gap factor $g_{\mathrm{c}}$ and the intermediate ring, whose width is defined by $\varepsilon$.

Maintaining the size of the PCFL (radius 12 $\mathrm{cm}$ ), when the width of the central gap and that of the lateral gap diminish, the fractal dimension $\mathrm{D}$ increases, causing the PCFL to become more opaque to the incident beam, then the diffraction, of each zone at stage 2, contributes to a focalization at different points. This fact produces a multi-foci profile in the nearfield. Therefore, when the fractal dimension of the designed lens, shown in Fig. $3 b$, acquires the value $\mathrm{D}=0.85$, the power of the focus that was located in $\mathrm{Z}=8 \lambda$ decreases, and the secondary approaches become more relevant. Therefore, the energy is distributed along the longitudinal axis $\mathrm{Z}$. The intermediate ring has a diffraction edge that contributes to the closest area of the PCFL. The numerical result corresponding to this case, is shown in Fig. $4 \mathrm{~b}$.

When the fractal dimension increases to the value $\mathrm{D}=0.90$, shown in Fig. $3 \mathrm{c}$, the width of the nearest transparent zones is less than $\lambda / 2$ of the signal. In this situation, the transmitted signal has been filtered and a well defined multi-focus has been achieved in the nearfield, as can be seen in the numerical result shown in Fig. 4c. As the central and lateral gaps of the PCFL are closed, a tendency to the periodicity of the transparent zones occurs, producing a change in the way in which the PCFL modulated the ultrasonic beam emitted by the piston. It is important to highlight it, the effect is similar to those in optics and signal processing with the phase apodization of aperture.

The numerical results obtained with FEM have been reproduced in controlled conditions following the set-up explained above. The figures $4 \mathrm{~d}, 4 \mathrm{e}$ and $4 \mathrm{f}$ show the results obtained for the three cases analyzed in numerical form: $\mathrm{D}=$ $0.80,0.85$ and 0.90 , respectively. This experimental set up have been used successfully, providing experimental results in good agreement with the 
numerical or analytical predictions obtained using the piston wave. The numerical simulations presented, supported by accurate experimental results allow us to validate the model.

\section{Conclusions}

In conclusion, three lenses based on Polyadic Cantor sets with three different fractal dimensions have been implemented. In addition, numerical models based on FEM have been validated. The results are in quite good agreement. PCFLs allows multi-foci beam profile with focal length variations depending on the fractal dimension. One of the benefits of these lenses is that can be built to work under several frequencies, other flat lenses are design and built for a single frequency. If the frequency is increased holding $D$, the focus shifts away. Such structures can be used in acoustics as phase apodization structures are used in optics and signal processing. In such a way, this type of lens construction opens another typological domain of flat lenses in the ultrasonic field for applications such as biomedical engineering.

\section{Acknowledgments}

Investigation supported by the Ministry of Economy, Industry and Competitiveness, and the European Regional Development Fund TEC201570939-R (MINECO/FEDER).

\section{References}

[1] O'Shea DC. Scalar Diffraction Theory. Diffractive optics: design, fabrication, and test. 2004;p. 17-35.

[2] Ge Y, Sun HX, Liu C, Qian J, Yuan SQ, Xia JP, et al. Acoustic focusing by an array of heat sources in air. Applied Physics Express. 2016;9(6).

[3] Xia JP, Sun HX, Cheng Q, Xu Z, Chen H, Yuan SQ, et al. Theoretical and experimental verification of acoustic focusing in metal cylinder structure. Applied Physics Express. 2016;9(5).

[4] Smit MK. New focusing and dispersive planar component based on an optical phased array. Electronics Letters. 1988;24(7):385.

[5] Jahns J, Huang A. Planar integration of free-space optical components. Appl Opt. 1989;28(9):16021605.
[6] Aieta F, Genevet P, Kats MA, Yu N, Blanchard R, Gaburro Z, et al. Aberration-free ultrathin flat lenses and axicons at telecom wavelengths based on plasmonic metasurfaces. Nano Letters. 2012;12(9):49324936.

[7] Calvo DC, Thangawng AL, Nicholas M, Layman CN. Thin Fresnel zone plate lenses for focusing underwater sound. Applied Physics Letters. 2015;107(1).

[8] Minin IV, Minin OV. 3D High-Quality Ultrasonic Imaging. In: Ultrasound Imaging-Medical Applications. InTech; 2011. .

[9] Mandelbrot BB. The fractal geometry of nature. vol. 1. WH freeman New York; 1982.

[10] Song GY, Huang B, Dong HY, Cheng Q, Cui TJ. Broadband focusing acoustic lens based on fractal metamaterials. Scientific reports. 2016;6:35929.

[11] Jaggard AD, Jaggard DL. Scattering from fractal superlattices with variable lacunarity. Journal of the Optical Society of America A. 1998;15(6):1626.

[12] Jaggard DL, Jaggard AD, Frangos PV. Fractal electrodynamics: Surfaces and superlattices. Frontiers in electromagnetics. 2000;709:1-47.

[13] Saavedra G, Furlan WD, Monsoriu JA. Fractal zone plates. Optics letters. 2003;28(12):971-973.

[14] Castiñeira-Ibáñez S, Rubio C, Redondo J, SánchezPérez JV. Quantitative characterization of bandgap properties of sets of isolated acoustic scatterers arranged using fractal geometries. Applied Physics Express. 2014;7(4).

[15] Castiñeira-Ibáñez S, Romero-García V, SánchezPérez JV, Garcia-Raffi LM. Overlapping of acoustic bandgaps using fractal geometries. EPL. 2010;92(2).

[16] Castiñeira-Ibáñez S, Tarrazó-Serrano D, Fuster J, Candelas P, Rubio C. Polyadic Cantor Fractal Ultrasonic Lenses: Design and Characterization. Applied Sciences. 2018;8(8):1389.

[17] Castiñeira-Ibáñez S, Tarrazó-Serrano D, Fuster JM, Candelas P, Rubio C. Polyadic Cantor Fractals: Characterization, Generation, and Application as Ultrasonic Lenses. In: Fractal Analysis - Applications in Health Sciences and Social Sciences. InTech; 2017. p. 88-107.

[18] Zienkiewicz OC, Taylor RL. The Finite Element Method Volume. In: Methods. vol. 1; 2000. p. 708.

[19] Castiñeira-Ibáñez S, Tarrazó-Serrano D, Rubio C, Candelas P, Uris A. An ultrasonic lens design based on prefractal structures. Symmetry. 2016;8(4):28.

[20] Rubio C, Fuster JM, Castiñeira-Ibáñez S, Uris A, Belmar F, Candelas P. Pinhole zone plate lens for ultrasound focusing. Sensors (Switzerland). 2017;17(7).

[21] Gómez-Lozano V, Rubio C, Candelas P, Belmar F, Uris A. Geometrical isotropy in perforated plates with subwavelength holes decorated with Archimedean patterns. EPL. 2015;111(3). 

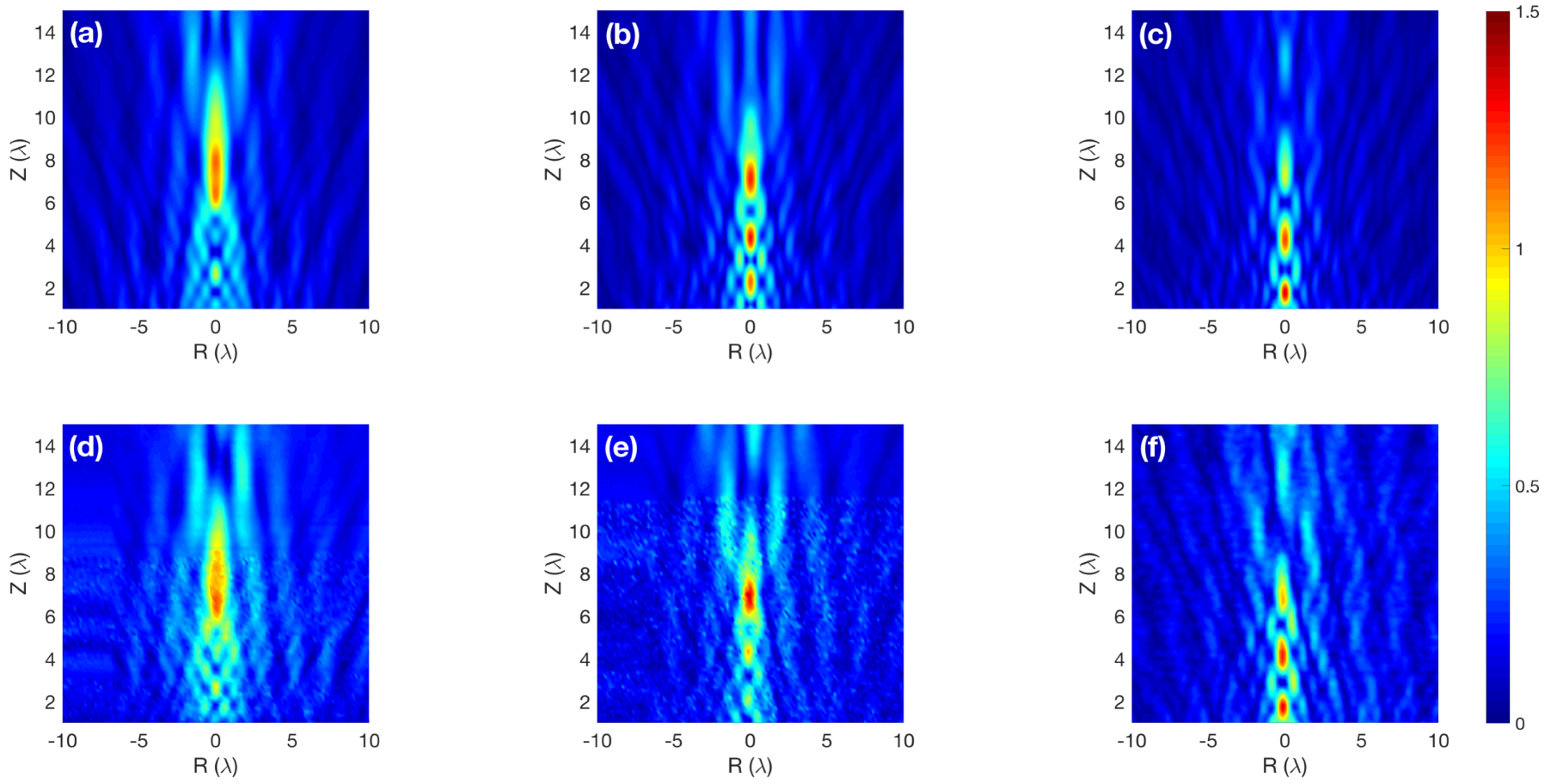

Figure 4: Absolute pressure maps normalized to $\lambda$ : Numerical solutions (a) $\mathrm{D}=0.80$, (b) $\mathrm{D}=0.85$, (c) $\mathrm{D}=0.90$. Experimental results: (d) $\mathrm{D}=0.80$, (e) $\mathrm{D}=0.85$, (f) $\mathrm{D}=0.90$ 